\title{
The Structural and Magnetic Properties of $\mathrm{CuRh}_{1-x} \mathrm{Mn}_{x} \mathrm{O}_{2}$ $(0 \leq x \leq 0.1)$ Delafossite Oxide
}

\author{
Taieb Elkhouni ${ }^{1}$ - M. Amami ${ }^{1,2}$ - E. K. Hlil ${ }^{3}$ A. Ben Salah ${ }^{1}$
}

Received: 25 October 2015 / Accepted: 16 December 2015 / Published online: 29 January 2016

(C) The Author(s) 2016. This article is published with open access at Springerlink.com

\begin{abstract}
The $\mathrm{CuRh}_{1-x} \mathrm{Mn}_{x} \mathrm{O}_{2}(0.00 \leq x \leq 0.10)$ polycrystalline powders were synthesized by the direct solidstate reaction. The magnetic susceptibility was measured in the temperature range of 4-300 K. It was found that the magnetic susceptibility $(\chi)$ increases rapidly with the doping of $\mathrm{Rh}^{3+}$ by $\mathrm{Mn}^{3+}$ ions with existence of paramagnetic substance at low temperature. For $x=0.1$, a development of new antiferromagnetic (AF) transition around 26 $\mathrm{K}$ and the magnetic data for $\mathrm{CuRh}_{1-x} \mathrm{Mn}_{x} \mathrm{O}_{2}$ show evidence for weak ferromagnetic (FM) transition between 100 and $130 \mathrm{~K}$. Clear hysteresis loops indicate that FM order exists in Mn-doped samples at $5 \mathrm{~K}$. All samples behave like semiconductors. The ferromagnetism properties can be attributed to the double-exchange interaction between the $\mathrm{Mn}^{3+}$ and $\mathrm{Rh}^{3+}$ semiconductor compounds. We analyze the magnetic entropy change $\Delta S_{\mathrm{M}}$ of $\mathrm{CuRh}_{0.9} \mathrm{Mn}_{0.1} \mathrm{O}_{2}$. Using Arrott plots, it was found that the phase transition for this sample at $T_{N}=42 \mathrm{~K}$ is of second order. The maximum entropy change of $\sim 0.017 \mathrm{~J} / \mathrm{kg} \mathrm{K}$ upon a filed variation of $\sim 1 \mathrm{~T}$ and $\sim 0.076 \mathrm{~J} / \mathrm{kg} \mathrm{K}$ upon a filed variation of $\sim 5$
\end{abstract}

Taieb Elkhouni

elkhouni@yahoo.fr

1 Laboratoire des Sciences de Matériaux et d'environnement, Faculté des Sciences de Sfax, BP 763, 3038 Sfax, Tunisie

2 Unité de Recherche de Chimie des Matériaux, ISSBAT, Université Tunis ElManar, 9, Avenue Dr. Zouhaier Safi, 1006 Tunis, Tunisie

3 Institut Néel, CNRS et Université Joseph Fourier, B.P. 166, Cedex 9, 38042 Grenoble, France
$\mathrm{T}$ was obtained, indicating relatively small magnetic order fluctuations around $T_{N}$.

Keywords Delafossite $\cdot$ Powder diffraction $\cdot$ Magnetic susceptibility $\cdot$ Antiferromagnetic transition $\cdot$ Magnetic entropy

\section{Introduction}

In recent years, there has been renewed interest in geometrically frustrated magnetic system. In such systems, spin ordering is suppressed by competing exchange interactions well below the conventional ordering scale set by the Weiss temperature. Consequently, they are expected to possess unconventional magnetic states, such as spin-liquid [1] and spin-nematic states [2]. $\mathrm{ABO}_{2}$-type materials, which have two-dimensional (2D) layered structures consisting of triangular lattices made up of magnetic B-site trivalent cations, have been studied as typical frustrated triangular-lattice spin systems [3]. In particular, $\mathrm{CuFeO}_{2}$ and $\mathrm{CuCrO}_{2}$ have been extensively studied in terms of magnetoelastic coupling and magnetoelectric multiferroics, which are considered to be associated with, spin frustration [4-7]. In other hands, geometrically frustrated magnetic systems have received a great deal of attention because their ordered or unordered ground states are often very exotic [1-3]. The triangular lattice antiferromagnet (TLA), in which magnetic ions reside at a triangular net, is the most obvious example of a geometrically frustrated magnetic system. A typical ordered structure in TLA is a noncollinear 3-sublattice $120 \circ$ spin configurations where the frustration of the three nearest-neighbor spins on a triangular plaquette is resolved by a $120 \circ$ rotation 
of neighboring spins. The $\mathrm{ABO}_{2}$ family with the delafossite structure ( $\mathrm{A}=$ monovalent ion, $\mathrm{B}=$ trivalent ion) has been recently investigated as one of the typical materials for the TLA.

In order to search for new thermoelectric materials, $\mathrm{CuRh}_{0.90} \mathrm{Mg}_{0.10} \mathrm{O}_{2}$ [8] has been investigated. One advantage of these materials over several others lies in their ability to be used at elevated temperatures in air. This opens the route to target systems releasing heat at temperatures as high as $1000 \mathrm{~K}$. Among the studied p-type thermoelectric oxides, the layered ones such as $\mathrm{CuRhO}_{2}$, or more recently the delafossites, all with structures containing CdI2-type layers have been particularly investigated according to the richness of their physical properties. For instance, the thermoelectric performance of several doped $\mathrm{CuRhO}_{2}$ delafossites have been measured, leading to the following dimensionless figures of merit $\mathrm{ZT}$ of 0.04 at $800 \mathrm{~K}\left(\mathrm{CuCr}_{0.97} \mathrm{Mg}_{0.03} \mathrm{O}_{2}\right)$ [8], 0.14 at $1100 \mathrm{~K}\left(\mathrm{CuFe}_{0.99} \mathrm{Ni}_{0.01} \mathrm{O}_{2}\right)$ [9], and 0.15 at 1000 $\mathrm{K}\left(\mathrm{CuRh}_{0.90} \mathrm{Mg}_{0.10} \mathrm{O}_{2}\right)$ [8]. Their crystal structure of the mother $\mathrm{CuRhO}_{2}$ can all be described as a delafossite-type oxide belongs to the space group R-3m. It has lattice parameters $a=3.0824 \AA$ and $c=17.0943 \AA$ [10]. It consists of a triangular lattice of $\mathrm{Rh}^{3+}$ ions $(S=0, L=0)$ separated by nonmagnetic ionic layers of $\mathrm{O}^{2-}, \mathrm{Cu}^{+}$, and $\mathrm{O}^{2-}$, stacked along the $c$ axis in the hexagonal description [11]. Among other magnetic delafossite, crednerite $\mathrm{CuMnO}_{2}$ is an $\mathrm{ABO}_{2}$-type triangular-lattice antiferromagnet that has $\mathrm{B}$ site cations of $\mathrm{Mn}^{3+}\left(3 \mathrm{~d}^{4}: \mathrm{t}_{2 \mathrm{~g}}^{3} \mathrm{e}_{\mathrm{g}}^{1}\right)$, the crystal structure of $\mathrm{CuMnO}_{2}$ does not consist of perfect triangular lattices [12], and rather, it consists of isosceles-triangular lattices due to Jahn-Teller distortion of $\mathrm{Mn}^{3+}\left(3 \mathrm{~d}^{4}: \mathrm{t}_{2 \mathrm{~g}}^{3} \mathrm{e}_{\mathrm{g}}^{1}\right)$ with an orbital degree of freedom. The crystal structure of $\mathrm{CuMnO}_{2}$ belongs to the monoclinic space group $\mathrm{C} 2 / \mathrm{m}$ at room temperature and it has lattice constants of $a=5.596 \AA, c=$ $5.899 \AA$, and $\beta=104.02^{\circ}$ [12]. Doumerc et al. [13] performed magnetic susceptibility measurements of $\mathrm{CuMnO}_{2}$; the electronic configuration of $\mathrm{Mn}^{3+}$ was found to be the high spin state $(S=2)$ and Weiss temperature $\Theta$ is -450 $\mathrm{K}$ [13]. They also used Mossbauer spectroscopy to investigate $\mathrm{Fe}$-doped samples and found a $3 \mathrm{D}$ long-range magnetic ordering which exhibits the antiferromagnetic phase transition at relatively low temperature $T_{N}=64 \mathrm{~K}$. The further cooling produces the week ferromagnetic phase transition at $42 \mathrm{~K}$, which is reported by the previous bulk measurements [13]. In the intermediate temperature regions of $T_{N}$ $<T<\Theta$, the susceptibility shows broad peaks reflecting the low-dimensional character. So far, the microscopic magnetic correlations and the dimensionality have not been clarified.

In this paper, we discuss another intriguing aspect, namely multiferroic nature, of geometrical spin frustration in a TLA. Recently, there has been a revival of interest in research of multiferroics showing the coexistence and/or interplay of magnetism and ferroelectricity socalled magnetoelectric (ME) effects [14, 15]. Among multiferroics studied to date, some exhibit strong couplings between magnetism and ferroelectricity, since the geometrical frustration in the magnetic triangular sublattice at the $\mathrm{M}$ sites causes intriguing properties, such as field induced multistep magnetization change [16] and multiferroics [17]. The introduction of charge carriers, which is possible in some delafossite compounds, may further induce novel phenomena arising from the coupling between the magnetic and transport properties in the quasi-two-dimensional frustrated triangular lattice. The magnetic degeneracy was lifted and the no collinear magnetic structure was realized in the antiferromagnetic (AF) regime below the ordering temperature $\left(T_{N}\right)$ to resolve the magnetic frustration $[18,19]$. This magnetic frustration appeared to be removed by the introduction of some trivalent cations in M-sites. In this study, to clarify in depth such a unique Mn-substitution effect, we investigated in detail the effect of the substitution of $\mathrm{Mn}^{3+}$, for $\mathrm{Rh}^{3+}$ on their magnetic properties of $\mathrm{CuRhO}_{2}$

\section{Experimental Section}

Polycrystalline samples of $\mathrm{CuRh}_{1-x} \mathrm{Mn}_{x} \mathrm{O}_{2}$ for different composition around $10 \%$ were prepared using the standard solid-state reaction. Stoichiometric mixtures $(0.5 \mathrm{~g})$ of $\mathrm{Cu}_{2} \mathrm{O}, \mathrm{Rh}_{2} \mathrm{O}_{3}$, and $\mathrm{Mn}_{2} \mathrm{O}_{3}$ were ground and pressed in pellets. The samples were fired repeatedly in air at $1150 \circ \mathrm{C}$ for $12 \mathrm{~h}$ in alumina crucibles with intermediate re-grinding and pelletizing. X-ray powder diffraction patterns of the products were collected with a PANalytical diffractometer equipped with a $\mathrm{CuK}_{\mathrm{a}}$ source $\left(\mathrm{K}_{\alpha 1}\right.$ and $\left.\mathrm{K}_{\alpha 2}\right)$ in the $2 \theta$ range from $10 \circ$ to $90 \circ$ at room temperature.

Magnetic susceptibilities measurements vs. temperature were carried out in a Quantum Design superconducting quantum interference device (SQUID) magnetometer in the range 4-300 $\mathrm{K}$. The typical field used for temperature dependence measurements was $0.1 \mathrm{~T}$. Magnetization measurements M vs. H used for MCE studies were realized with the BS2 magnetometer developed at Néel Institute.

\section{Results and Discussion}

\subsection{Structural Properties}

The crystal structure of $\mathrm{CuCr}_{1-x} \mathrm{Mn}_{x} \mathrm{O}_{2}(0 \leq x \leq 0.1)$ samples is determined by recording $\mathrm{X}$-ray diffraction patterns 
Fig. 1 Room temperature XRD patterns of $\mathrm{CuRh}_{1-x} \mathrm{Mn}_{x} \mathrm{O}_{2}$ $(0.00 \leq x \leq 0.10)$ samples with a rhombohedral delafossite structure

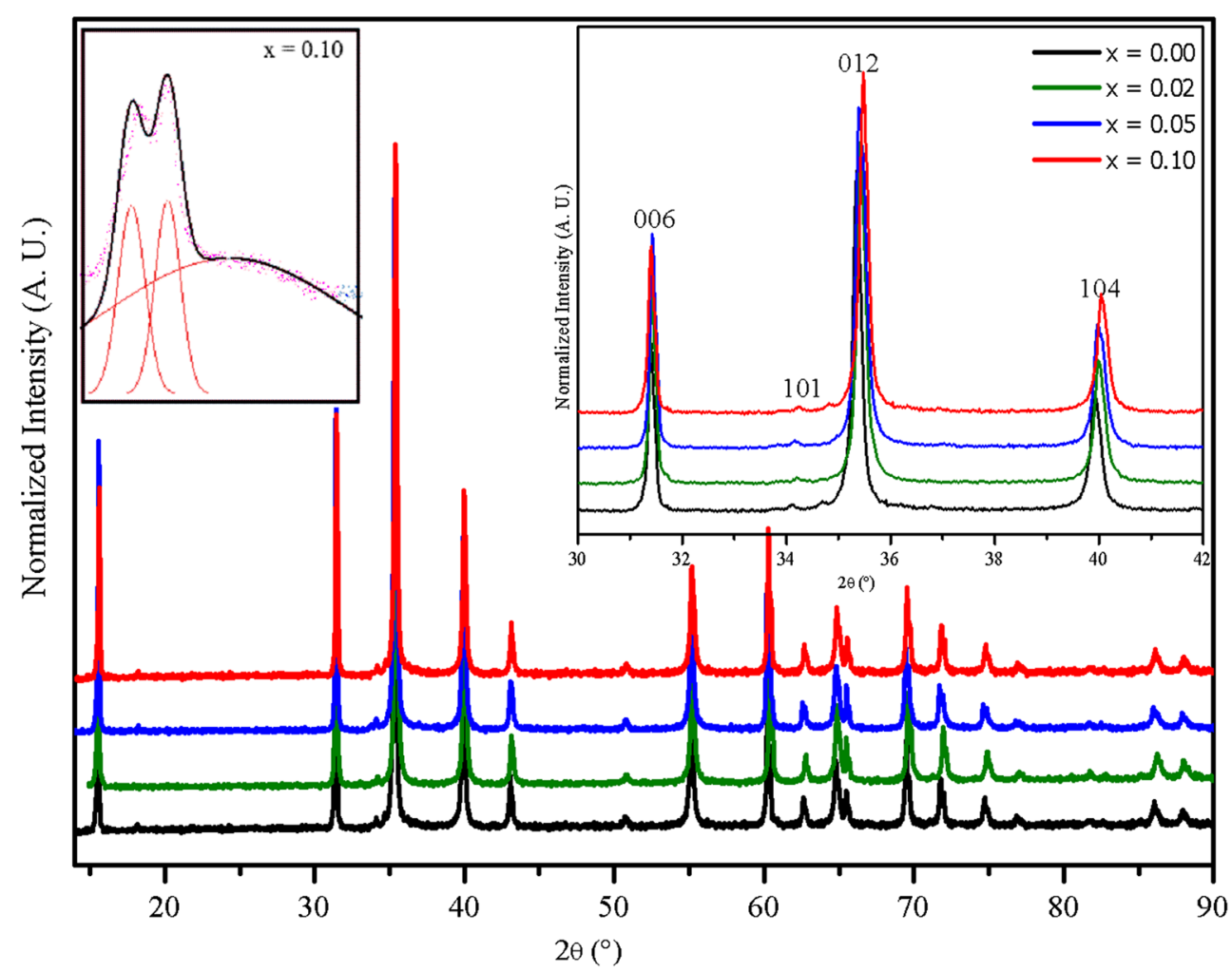

shown in Fig. 1. According to the standard XRD patterns of $\mathrm{CuRhO}_{2}$, samples are of single phase with delafossite structure (space group R-3m), without any detectable secondary phase up to $10 \% \mathrm{Mn}$ content. For this composition, the occurrence of structural transformation is clearly demonstrated in the inset of Fig. 1 by the splitting of the (012) Bragg peak into two components which are identified by a decrease of the parent rhombohedral phase and a development of new crednerite $\mathrm{CuMnO}_{2}$ phase with increasing of $\mathrm{Mn}$ content. In fact, we conclude that the delafossite $\mathrm{CuRhO}_{2}$ tend to be Crednerite $\mathrm{CuMnO}_{2}$ at $10 \%$ of $\mathrm{Mn}$ substitution.

Similar to previous observation by Damy et al. [20], the room temperature of crystal structure of $\mathrm{CuRh}_{1-x} \mathrm{Mn}_{x} \mathrm{O}_{2}$ $0.00 \leq x \leq 0.1$ compounds was found to be rhombohedral with a slight decreases of the $a$ lattice constant. This small compression of the oxygen framework can be accommodated in a psedomonoclinic $\mathrm{C} 2 / \mathrm{m}$ symmetry, which maintains a similar description of the high-content crystal lattice. $\mathrm{The} \mathrm{Rh} / \mathrm{MnO}_{6}$ octahedra undergo a small compression in its equatorial plane, which produces a decrease of Rh-Mn bond with increase of $\mathrm{x}$ content.

Composition dependences of the unit cell volume and lattice constants are given in Table 1. For $x=0$ (pure $\mathrm{CuRhO}_{2}$ ), our $a$ and $c$ parameter values are in very good agreement with most of those previously reported for powders as well as for single crystal (e.g. $a=3.082(3) \AA, c=$ 17.094(3) Å from [10]).

The decreases of the unit cell volume with increasing $x$ well agrees with the decrease of the ionic radius as rhodium $(r=0.66 \AA)$ is substituted for manganese ( $r=$ $0.58 \AA$ ) [21]. However, the change is rather anisotropic as it is mainly due to the shrinking of the $a$ parameter whereas the $c$ parameter remains more or less constant.

As the $\mathrm{Cu}-\mathrm{O}$ distance does not vary much in delafossite, this tendency reflects a flattening of the $\mathrm{MO}_{6}$ octahedra. A nice geometric account of this evolution is given by J. Tate et al. [22] where the authors suggest that the strong repulsion between $\mathrm{M}^{3+}$ ions across the octahedron shared edges reduces the $\mathrm{O}-\mathrm{O}$ distance to the contact distance.

Therefore, the decreases of the size of $\mathrm{M}$ cation leads to a decrease of the octahedron distortion and in turn of the

Table 1 The lattice parameters $a, c$, and cell volumes $V$ of the $\mathrm{CuRh}_{1-x} \mathrm{Mn}_{x} \mathrm{O}_{2}(0.00 \leq x \leq 0.10)$ compounds

\begin{tabular}{llll}
\hline Samples & $a(\AA)$ & $c(\AA)$ & $V\left(\AA^{3}\right)$ \\
\hline $\mathrm{CuRhO}_{2}$ & $3.082(3)$ & $17.094(3)$ & $140.656(5)$ \\
$\mathrm{CuRh}_{0.98} \mathrm{Mn}_{0.02} \mathrm{O}_{2}$ & $3.079(6)$ & $17.093(1)$ & $140.391(2)$ \\
$\mathrm{CuRh}_{0.95} \mathrm{Mn}_{0.05} \mathrm{O}_{2}$ & $3.075(1)$ & $17.092(8)$ & $139.978(8)$ \\
$\mathrm{CuRh}_{0.90} \mathrm{Mn}_{0.10} \mathrm{O}_{2}$ & $3.059(3)$ & $17.092(5)$ & $138.541(6)$ \\
\hline
\end{tabular}


M-M distance that corresponds to the aparameter. We may recall that in $\mathrm{CuMO}_{2}$, as $\mathrm{M}$ changes from $\mathrm{La}^{3+}$ to $\mathrm{Al}^{3+}$ $[23,24]$, the aparameter undergoes a huge decreases from 3.8 up to $2.8 \AA$.

\subsection{Magnetic Properties}

Figure 2 shows the temperature dependence of magnetic susceptibility of $\mathrm{CuRh}_{1-x} \mathrm{Mn}_{x} \mathrm{O}_{2}(0.00 \leq x \leq 0.10)$ samples at $0.1 \mathrm{~T}$. The $\chi(T)$ curves of the parent delafossite $\mathrm{CuRhO}_{2}$, a paramagnetic comportment in the temperature range 4 to $300 \mathrm{~K}$, for the content $x=0.02$ and $x=0.05$ the magnetic susceptibility present a short increases of the magnetic susceptibilities with increases of $x$ content at height temperature. The $\chi(T)$ curve of the $\mathrm{CuRh}_{0.9} \mathrm{Mn}_{0.10} \mathrm{O}_{2}$ sample showed a sharp transition at $T_{N}=42 \mathrm{~K}$ from a paramagnetic to antiferromagnetic state followed by an increases of the magnetic susceptibility at height temperature.

This behavior of the $\chi(T)$ curves for $\mathrm{CuRh}_{1-x} \mathrm{Mn}_{x} \mathrm{O}_{2}$ series at height temperature region agrees with results shown in [10]. In fact, in this reference, it has been reported that the increase in the magnetic susceptibility in $\mathrm{CuRh}_{1-x} \mathrm{Co}_{x} \mathrm{O}_{2}$ is significant only with increasing of $x$ content.

The replacement of $\mathrm{Rh}^{3+}$ by $\mathrm{Mn}^{3+}$ develop some ratios of $\mathrm{Mn}^{3+}-\mathrm{O}^{2-}-\mathrm{Mn}^{4+}$ bonds and the interactions between $\mathrm{Rh}-\mathrm{O}-\mathrm{Mn}$ might be an antiferromagnetic super-exchange. The competition between the $\mathrm{Mn}-\mathrm{O}-\mathrm{Mn}$ bonds are doubleexchange (ferromagnetic) and $\mathrm{Rh}-\mathrm{O}-\mathrm{Mn}$ super-exchange (antiferromagnetic) may explain the weakening increase of the magnetic susceptibility and the development of the ferromagnetism with appears of new crednerite phase $\mathrm{CuMnO}_{2}$ in our substituted sample $x=0.1$. All those results are in a good agreement with those reported by $\mathrm{S}$. Hebert et al. [25] which reported that the substitution in Mnsites of the mother compound $\mathrm{LaMnO}_{3}$ induce disorder on the manganese site, and prevent the establishment of conducting paths along the $\mathrm{Mn}-\mathrm{O}-\mathrm{Mn}$ bonds. Conversely, the introduction of trivalent cations does not induce a strong ferromagnetism and the electronic transport is obviously hindered by these foreign cations on the manganese site. This supposition explain the strong ferromagnetic transition in $\mathrm{LaMnO}_{3}$ which is induced by introducing mixed-valent manganese $\mathrm{Mn}^{3+} / \mathrm{Mn}^{4+}$ and they induce disorder on the $\mathrm{Mn}$-sites. These phenomena explain the variation of the amplitude transition ferro-paramagnetic with the temperature.

The inverse of the susceptibility, $\chi^{-1}$, versus temperature curves are plotted in inset of Fig. 2. Those plots can be described in the paramagnetic region by a CurieWeiss law, defined by the relation $\chi=\chi_{0}+C /(T-\Theta)$ [10], where $C$ is the Curie constant and $\Theta$ is the asymptotic Curie temperature. By fitting the linear paramagnetic region, the Curie-Weiss $C$ and $\Theta$ parameters were obtained (Table 2). The negative values of @indicate the strong antiferromagnetic interactions resulting from the Mn substitution. Moreover, the increase of $\Theta$ value and the convergence of those values to positive prove that those interactions converge to ferromagnetic state between spin. Correlating the magnetic properties in $\mathrm{CuRh}_{1-x} \mathrm{Mn}_{x} \mathrm{O}_{2}$ system with those
Fig. 2 Temperature $(T)$ dependence of zero-fieldcooling (ZFC) susceptibility of $\mathrm{CuRh}_{1-x} \mathrm{Mn}_{x} \mathrm{O}_{2}$ for $0.00 \leq x \leq$ 0.10 at $0.1 \mathrm{~T}$. Inset, temperature $(T)$ dependence of the inverse susceptibility

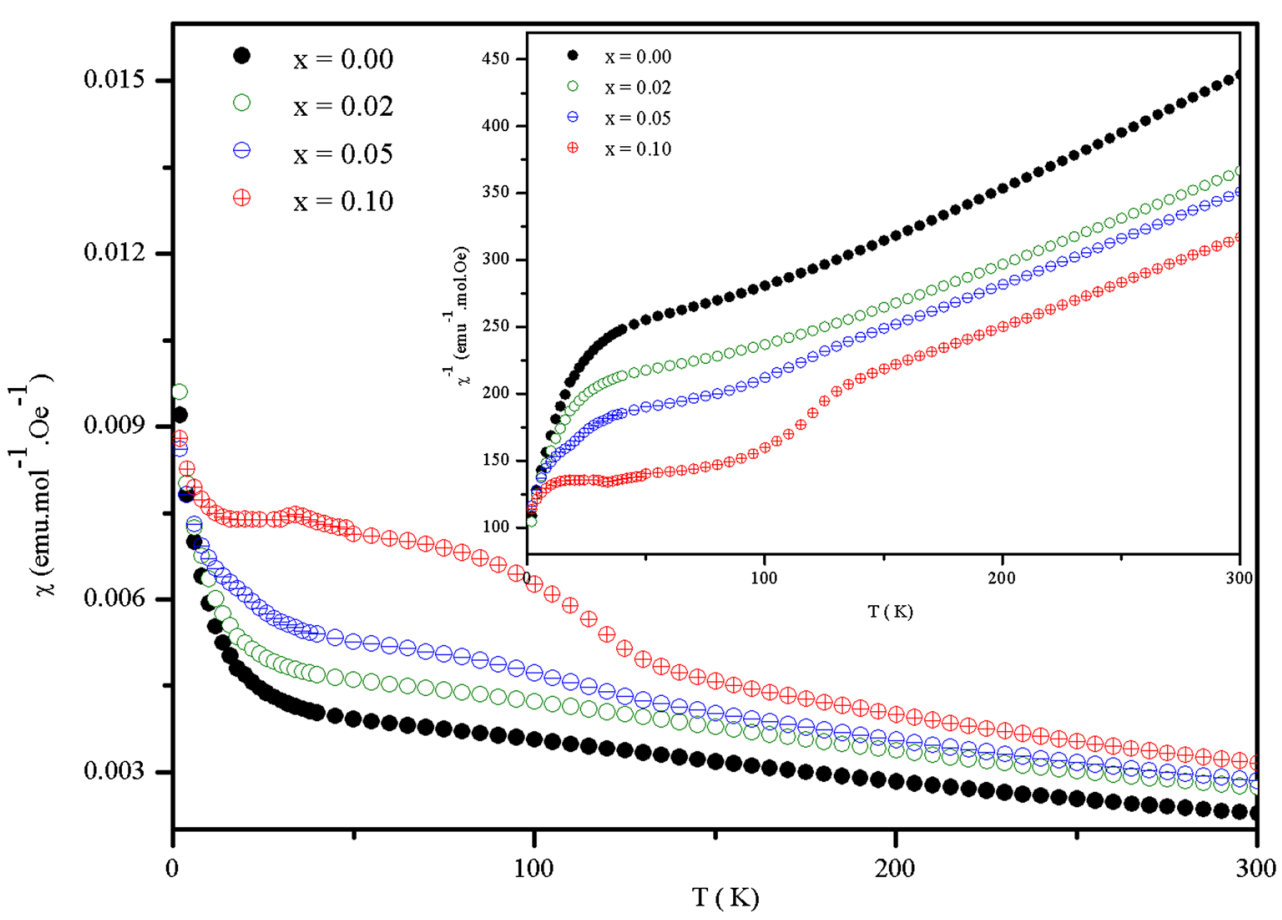


Table 2 Estimated Curie constant Curie temperature and effective magnetic moment from the hightemperature paramagnetic region for $\mathrm{CuRh}_{1-x} \mathrm{Mn}_{x} \mathrm{O}_{2}(0.00 \leq x \leq$ 0.10)

\begin{tabular}{lllll}
\hline Samples & $\mathrm{CuRhO}_{2}$ & $2 \%$ & $5 \%$ & $10 \%$ \\
\hline$\chi_{0}\left(\mathrm{emu} \mathrm{mol}^{-1} \mathrm{Oe}^{-1}\right)$ & $1.6 \mathrm{E}^{-3}$ & $4.6 \mathrm{E}^{-3}$ & $9.2 \mathrm{E}^{-4}$ & $11.2 \mathrm{E}^{-4}$ \\
$C(\mathrm{emu} \mathrm{K} \mathrm{mol}$ & -1 & $0.0068(8)$ & $0.0084(3)$ & $0.0102(7)$ \\
$\Theta(\mathrm{K})$ & $0.0048(4)$ & $-75.3(2)$ & $-52.5(1)$ & $-27.1(7)$ \\
$\mu_{\mathrm{eff}}\left(\mu_{\mathrm{B}}\right)$ & $-81.6(4)$ & $0.2346(1)$ & $0.2596(9)$ & $0.2866(3)$ \\
\hline
\end{tabular}

of structural properties. The origin of increasing magnetic susceptibility is proved by the development of new phase which is deconvoluted by the development of new crednerite $\mathrm{CuMnO}_{2}$ phase. From the magnetic properties, the tendency to the ferromagnetic state proves those structural propositions.

In order to investigate the magnetic behavior of the system $\mathrm{CuRh}_{1-x} \mathrm{Mn}_{x} \mathrm{O}_{2}$, we have carried out magnetic susceptibilities versus the magnetic applied field at height temperature $(\chi(H)$ at 5 and $300 \mathrm{~K})$ as shown in Fig. 3a, $\mathrm{b}$, respectively. We can distinguish two different types of the $\chi-H$ curves as a function of manganese content. For all samples, we observe a typical behavior of antiferromagnetic states, when the magnetic susceptibilities at low temperatures $(5 \mathrm{~K})$ increase sharply and then saturates as shown in Fig. 3a. This antiferromagnetic results show that the Mn substitutions strongly weaken the antiferromagnetic and stabilize this phase.

The height temperature $(300 \mathrm{~K}) \chi-H$ curves for $\mathrm{CuRh}_{0.9} \mathrm{Mn}_{0.1} \mathrm{O}_{2}$ ceramic is presented in Fig. 3b. For a closer observation, the curve is magnified partially. It is seen that the $\chi-H$ loop apparently shows hysteresis, indicating ferromagnetism of the $\mathrm{Mn}$-doped $\mathrm{CuRhO}_{2}$ sample. Also, a linear paramagnetic behavior superimposed upon hysteresis is observed, which may originate from paramagnetic regions contained in the samples due to nearly isolated $\mathrm{Mn}$ ions, e.g., where the local Mn concentration is the lowest, this results is in a good agreement with those reported by $\mathrm{C}$. Gao et al. [26] for Fe substituted $\mathrm{CuCrO}_{2}$.

The disorder of the $\mathrm{Rh}$ and Mn cations complicates the interpretation of the magnetic properties. The interaction of the $\mathrm{M}$ cations with each other through $\mathrm{Rh}-\mathrm{O}-\mathrm{Mn}$ linkages of approximately $180 \circ$ is expected to be the dominant consideration, and this would be antiferromagnetic in nature. Recently, it was reported on evidence of room temperature ferromagnetism in Co-doped transparent $\mathrm{CuAlO}_{2}$ semiconductor [27]. Specially, the coercively ( $\mathrm{Hc})$ and saturation magnetization are significantly enhanced with Co composition [27]. In Fig. 3b and at $300 \mathrm{~K}$, a remarkable loop for $\mathrm{CuRh}_{0.9} \mathrm{Mn}_{0.1} \mathrm{O}_{2}$ is observed, giving rise such us to a coercivity of 4000 Oe and remnant magnetization of $\sim 1.3$ $10^{-4}$ emu $\mathrm{mol}^{-1}$, although no magnetization saturation is obtained, probably due to the spin-glasslike essence. Therefore, the substantial ferromagnetic crednerite component $\mathrm{CuMnO}_{2}$ in the magnetism of $\mathrm{CuRh}_{1-x} \mathrm{Mn}_{x} \mathrm{O}_{2}$ is revealed.
The observed magnetic behavior for $\mathrm{CuRh}_{1-x} \mathrm{Mn}_{x} \mathrm{O}_{2}$ is probably due to the disorder of $\mathrm{Rh}$ and $\mathrm{Mn}$ in octahedral sites resulting in short-range $\mathrm{Rh}-\mathrm{O}-\mathrm{Rh}$ and $\mathrm{Mn}-\mathrm{O}-\mathrm{Mn}$ interactions between which could give rise to antiferromagnetism coupled with short-range weak ferromagnetism. Thus, the observed ferromagnetism is essentially intrinsic to some extent. The origin of ferromagnetism in DMSs is still not very clear, although many efforts have been made to explain it. It is believed from the calculated density of states (DOS) that the double-exchange interaction is the dominant exchange mechanism in TM-doped $\mathrm{CuAlO}_{2}$ based DMSs [28]. It is also found that indirect interaction among Co centers results in ferromagnetism, whereas direct interaction among them induces antiferromagnetism [29, 30].

\subsection{Magnetic Entropy}

In order to investigate the change in magnetic entropy, isothermal magnetization curves are recorded around $T_{N}$ for the sample $\mathrm{CuRh}_{0.9} \mathrm{Mn}_{0.1} \mathrm{O}_{2}$ in a magnetic field up to $5 \mathrm{~T}$. Figure $4 \mathrm{a}$ shows the measured isothermal magnetization for the considered sample in the form of polynomial fitting of $H / M$ vs. $M^{2}$ Arrott plots. A more standard type of Arrott plots is observed. The plots display the nonlinear behavior, and the positive slope is clearly seen in the complete $M^{2}$ range.

According to the criterion proposed by Banerjee [31], all the properties of the Arrott plots show characteristics of second-order magnetic phase transition. The magnetocaloric effect is calculated indirectly with Maxwell's relation from the magnetization data in various applied magnetic field [32] from 0 to $\mu_{0} H_{\max }$ as follows:

$$
\begin{aligned}
\Delta S_{M}\left(T, \Delta\left(\mu_{0} H\right)\right) & =S\left(T, \mu_{0} H_{1}\right) \\
& =\int_{\mu_{0} H_{1}}^{\mu_{0} H_{2}}\left(\frac{\delta M\left(T, \mu_{0} H\right)}{\delta T}\right) d\left(\mu_{0} H\right)
\end{aligned}
$$

It could be written as follows:

$$
\begin{aligned}
\Delta S_{M}\left(\frac{T_{1}+T_{2}}{2}\right)= & \frac{1}{T_{2}-T_{1}}\left[\int_{0}^{\mu_{0} H} M\left(T_{2}, \mu_{0} H\right) d\left(\mu_{0} H\right)\right. \\
& \left.-\int_{0}^{\mu_{0} H} M\left(T_{1}, \mu_{0} H\right) d\left(\mu_{0} H\right)\right]
\end{aligned}
$$


where $(\partial M / \partial T)_{\mu_{0} H_{i}}$ is the experimental value obtained from $M-T$ curve in a magnetic field $\mu_{0} H_{i}$.

Figure $4 \mathrm{~b}$ presents the magnetic entropy change $\Delta S_{M}$ for the sample $\mathrm{CuRh}_{0.9} \mathrm{Mn}_{0.1} \mathrm{O}_{2}$ as a function of temperature under different magnetic fields. A remarkable feature is the exact correspondence between the maximal $\Delta S_{M}$ and the recorded PM-AFM transition at $T_{N}$, while $\Delta S_{M}$ distributes over a relatively wide $T$ range. The maximum entropy change of $\sim 0.017 \mathrm{~J} / \mathrm{kg} \mathrm{K}$ upon a filed variation of $\sim 1 \mathrm{~T}$ and $\sim 0.076 \mathrm{~J} / \mathrm{kg} \mathrm{K}$ upon a filed variation of $\sim 5 \mathrm{~T}$ was obtained, indicating relatively small magnetic order fluctuations around $T_{N}$. This result proves the idea of existence of weak antiferromagnetic interaction at low temperatures.

\section{Discussion}

The discussion above comes to a conclusion that the observed ferromagnetic behavior should be an intrinsic property of $\mathrm{Mn}$-substituted $\mathrm{CuRhO}_{2}$ solid reaction. According to refs. [33-36], the $\mathrm{Mn}^{3+}$ subsisting can destabilize the anti-ferromagnetic order of $\mathrm{Rh}^{3+}$ ions, modulate the spin configuration, and bring about ferromagnetism. Although the super-exchange interaction between $\mathrm{Mn}^{3+}$ is also antiferromagnetic [37], the coexistence of $\mathrm{Mn}^{3+}$ and $\mathrm{Rh}^{3+}$ should induce a new magnetic exchange mechanism. The single valence state of $\mathrm{Mn}^{3+}$ and $\mathrm{Rh}^{3+}$, revealed by the XRD results, excludes the possibility of double-exchange
Fig. 3 a Measured $\chi-H$ hysteresis at $5 \mathrm{~K}$ for $\mathrm{CuRh}_{1-x} \mathrm{Mn}_{x} \mathrm{O}_{2}(0 \leq x \leq$ 0.10). b Measured $\chi-H$ hysteresis at $300 \mathrm{~K}$ for $\mathrm{CuRh}_{0.9} \mathrm{Mn}_{0.1} \mathrm{O}_{2}$
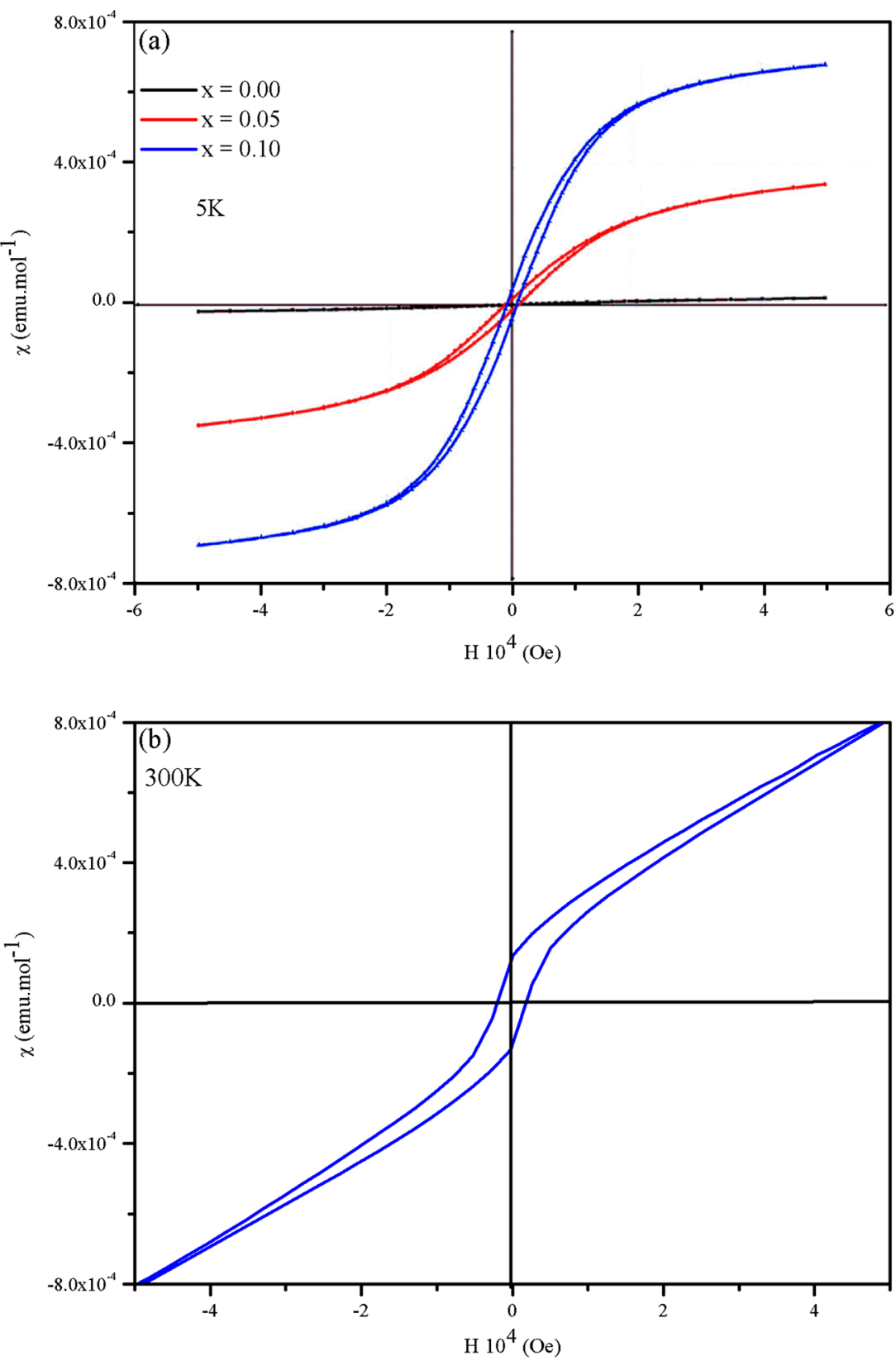
Fig. $4 H / M$ vs. $M^{2}$ (a) isotherms for $\mathrm{CuRh}_{0.9} \mathrm{Mn}_{0.1} \mathrm{O}_{2}$. The temperatures of the isotherms are indicated. Magnetic entropy change $\left(-\Delta S_{M}\right)(\mathbf{b})$ as a function of temperature in various magnetic fields up to $5 \mathrm{~T}$
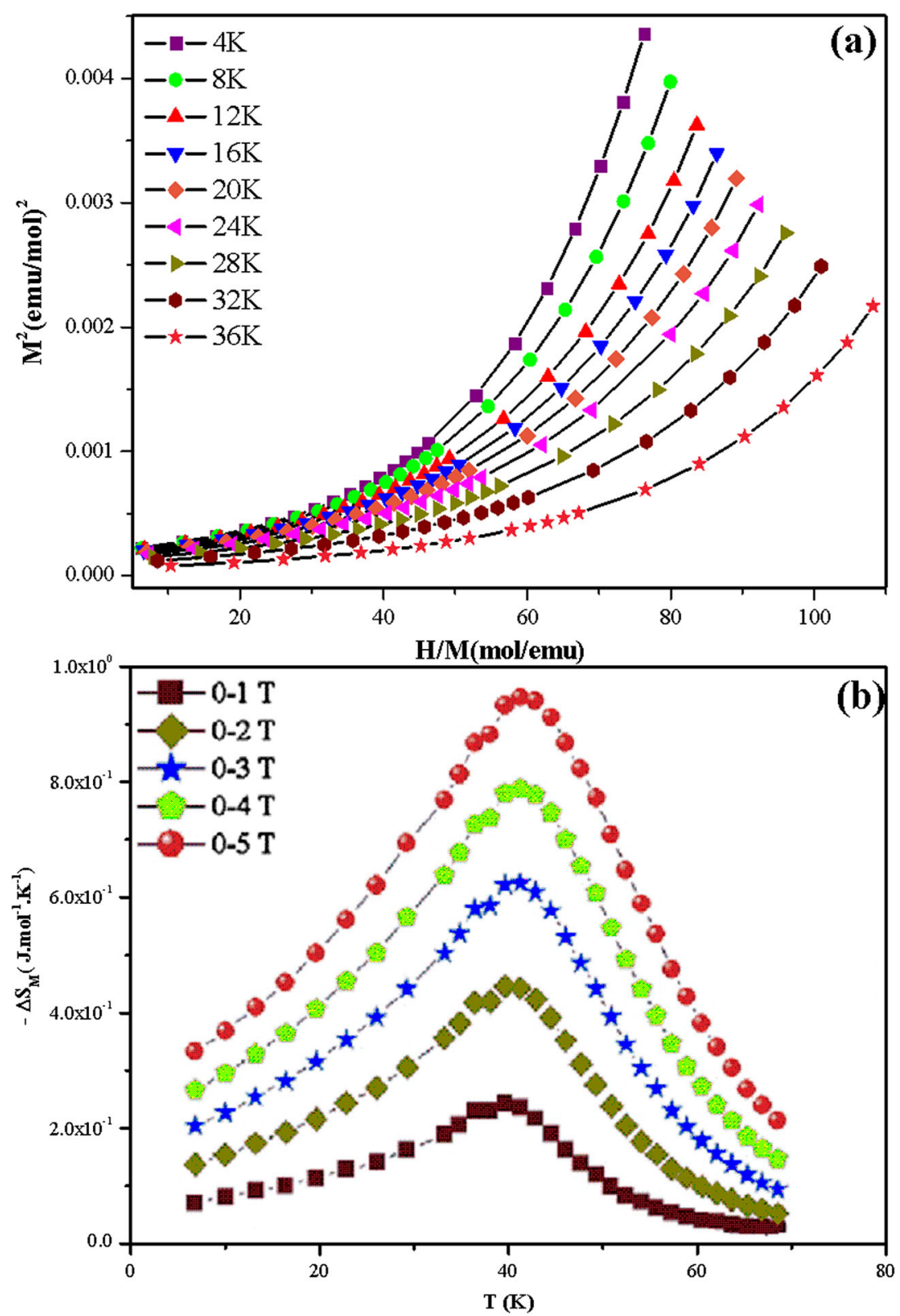

mechanism. Hence, the hole-mediated $\mathrm{Mn}^{3+}-\mathrm{Rh}^{3+}$ superexchange interaction can be expected to produce ferromagnetism which overcomes the anti-ferromagnetic $\mathrm{Rh}^{3+}{ }_{-}$ $\mathrm{Rh}^{3+}$ and $\mathrm{Mn}^{3+}-\mathrm{Mn}^{3+}$ couplings. With the increasing $\mathrm{Mn}$ concentration, both the number of the $\mathrm{Mn}^{3+}-\mathrm{Rh}^{3+}$ pairs and the hole concentration are enhanced. Accordingly, the hole-mediated $\mathrm{Mn}^{3+}-\mathrm{Rh}^{3+}$ ferromagnetic coupling is strengthened, leading to the gradual increase in both $M_{S}$ and $\Theta$.

The $\chi-T$ curves of $\mathrm{CuRh}_{1-x} \mathrm{Mn}_{x} \mathrm{O}_{2}$ ceramics were measured by SQUID at a magnetic field of $0.1 \mathrm{~T}$. The entire Mn-doped samples exhibit greatly enhanced the magnetic effective moment $\left(\mu_{\mathrm{eff}}\left(\mu_{\mathrm{B}}\right)\right)$, compared with literature values. With the change of Mn concentration, the $\mu_{\mathrm{eff}}\left(\mu_{\mathrm{B}}\right)$ values range between $\sim 0.196 \mu_{\mathrm{B}}$ and $\sim 0.286 \mu_{\mathrm{B}}$. Its $\mu_{\mathrm{eff}}\left(\mu_{\mathrm{B}}\right)$ can be evaluated as much less than those of $\mathrm{CuCr}_{1-x} \mathrm{Mn}_{x} \mathrm{O}_{2}$ ceramics [38] $(x \geq 0)$. The $\chi-T$ results also provide strong evidence that the ferromagnetism cannot come from delafossite $\mathrm{CuRh}_{1-x} \mathrm{Mn}_{x} \mathrm{O}_{2}$ but it is attributed to the development of new crednerite phase $\mathrm{CuMnO}_{2}$ which is identified by XRD pattern. This ferromagnetic impurity were present; it could not account for the $\mathrm{Mn}$ concentration dependence of magnetization. Note that increasing the Mn concentration leads to a gradual decrease in $M_{S}$. If manganese oxides were responsible for ferromagnetic behavior, the increase of $\mathrm{Mn}$ concentration would presumably enhance the corresponding phase volume fraction and related magnetization signature. Instead, 
the opposite behavior is observed. Therefore, the ferromagnetism is considered to be an intrinsic property of $\mathrm{Mn}$ doped $\mathrm{CuRhO}_{2}$ ceramics. According to ref. [38], Mn-doped $\mathrm{CuCrO}_{2}$ can destabilize the anti-ferromagnetic order of $\mathrm{Cr}^{3+}$ ions, whereas the super-exchange interaction between $\mathrm{Rh}^{3+}$ is also known to be anti-ferromagnetic [39]. Nevertheless, the coexistence of $\mathrm{Mn}^{3+}$ and $\mathrm{Rh}^{3+}$ should induce different magnetic exchange interaction. Z. Yang et al. [40] supposed that the magnetic interactions between $\mathrm{Mn}^{3+}$ and $\mathrm{Mn}^{3+}$ ions ${ }^{+}$are ferromagnetic; the magnetic interactions between $\mathrm{Rh}^{3+}$ and $\mathrm{Rh}^{3}$ ions are antiferromagnetic.

On the other hand, F. Dammy et al. [20] reported that the substitution in $\mathrm{Mn}$-sites of the mother compound $\mathrm{CuMnO}_{2}$ induce disorder on the manganese site, and prevent the establishment of conducting paths along the Mn-O-Mn bonds. Conversely, the introduction of trivalent cations does not induce a strong ferromagnetism and the electronic transport is obviously hindered by these foreign cations on the manganese site. This supposition explain the strong ferromagnetic transition in $\mathrm{CuMnO}_{2}$ which is induced by introducing mixed-valent manganese $\mathrm{Mn}^{3+} / \mathrm{Mn}^{4+}$ and they induce disorder on the Mn-sites. This mixture valence state of $\mathrm{Mn}^{3+} / \mathrm{Mn}^{4+}$ certified the possibility of double-exchange mechanism inducing three-dimensional ferromagnetic interactions in $\mathrm{CuMnO}_{2}$ compounds and shows a weak ferromagnetism.

Hence, the hole-mediated $\mathrm{Mn}^{3+}-\mathrm{Rh}^{3+}$ super-exchange interaction can be expected to produce ferromagnetism of $\mathrm{Mn}$-doped $\mathrm{CuRhO}_{2}$. The ferromagnetic $\mathrm{Mn}^{3+} / \mathrm{Mn}^{4+}-\mathrm{Rh}^{3+}$ coupling dominates over the crednerite phase $\mathrm{CuMnO}_{2}$, giving rise to the ferromagnetic behavior and enhanced $\Theta$.

As increasing the Mn concentration, both interactions $\mathrm{Mn}^{3+}-\mathrm{Rh}^{3+}$ and $\mathrm{Mn}^{4+}-\mathrm{Rh}^{3+}$ pairs are increased while the $\mathrm{Rh}^{3+}-\mathrm{Rh}^{3+}$ pairs are decreased. It means that ferromagnetic couplings would be strengthened whereas antiferromagnetic $\mathrm{Rh}^{3+}-\mathrm{Rh}^{3+}$ coupling just the reverse. Meanwhile, the magnetization has a high sensitivity to small changes in lattice constants [38]. For this $\mathrm{CuRh}_{1-x} \mathrm{Mn}_{x} \mathrm{O}_{2}$ structure, a decrease of the $a$ parameter (see Table 1) corresponds to short $\mathrm{Rh}-\mathrm{Mn}$ distances, which will influence the Rh-Mn magnetic interactions. Besides, the hole concentration may change with the Mn addition, which would also have an effect on the hole-mediated exchange interactions. The competitions among these three factors, that is, the number of the $\mathrm{Rh}-\mathrm{Mn}$ pairs, the $\mathrm{Rh}-\mathrm{Mn}$ distances, and the hole concentration, eventually result in an almost linear reduction of $\mathrm{M}_{\mathrm{S}}$ with the increasing $\mathrm{Mn}$ concentration.

\section{Conclusion}

In summary, we investigated the structural, magnetic properties and magnetocaloric effect of $\mathrm{CuRh}_{1-x} \mathrm{Mn}_{x} \mathrm{O}_{2}(0 \leq$ $x \leq 0.1)$. The structure of $\mathrm{CuRh}_{1-x} \mathrm{Mn}_{x} \mathrm{O}_{2}$ was delafossite phase for the studied $x$ range and a decrease of the lattice parameters $a$ among the samples. $\mathrm{CuRhO}_{2}$ is paramagnetic oxide; the magnetic susceptibilities increased with increasing Mn content, up $x=0.1$ which develops with new antiferromagnetic transition temperature $T_{N}=42 \mathrm{~K}$. The magnetization has a high sensitivity to small changes in lattice constants; for this $\mathrm{CuRh}_{1-x} \mathrm{Mn}_{x} \mathrm{O}_{2}$ structure, a decrease of the $a$ parameter corresponds to decreases of $\mathrm{Rh}-$ Mn distances, which will increase the Rh-Mn magnetic interactions to develop this new antiferromagnetic transition $T_{N}=$ $42 \mathrm{~K}$ indicating partial release of the spin frustration. The magnetocaloric effect for the sample $\mathrm{CuRh}_{0.9} \mathrm{Mn}_{0.1} \mathrm{O}_{2}$ has been investigated around $T_{N}$ in a magnetic field up to $5 \mathrm{~T}$. A maximum entropy $\Delta S_{\max }$ was obtained; it changed with $\sim 0.017 \mathrm{~J} / \mathrm{kg} \mathrm{K}$ upon a filed variation of $\sim 1 \mathrm{~T}$ and $\sim 0.076$ $\mathrm{J} / \mathrm{kg} \mathrm{K}$ upon a filed variation of $\sim 5 \mathrm{~T}$, indicating relatively small magnetic order fluctuations around $T_{N}$.

Acknowledgments This work was financially supported by Université Joseph Fourier (UJF Chimie), Grenoble, France.

Open Access This article is distributed under the terms of the Creative Commons Attribution 4.0 International License (http:// creativecommons.org/licenses/by/4.0/), which permits unrestricted use, distribution, and reproduction in any medium, provided you give appropriate credit to the original author(s) and the source, provide a link to the Creative Commons license, and indicate if changes were made.

\section{References}

1. Balents, L.: Nature (London) 464, 199 (2010)

2. Chandra, P., Coleman, P.: Phys. Rev. Lett. 64, 88 (1990)

3. Collins, M.F., Petrenko, O.A.: Can. J. Phys. 75, 605 (1997)

4. Terada, N., Narumi, Y., Sawai, Y., Katsumata, K., Staub, U., Tananka, Y., Kikkawa, A., Fukui, T., Kindo, K., Yamamoto, T., Kanmuri, R., Hagiwara, M., Toyokawa, H., Ishikawa, T., Kitamura, H.: Phys. Rev. B 75, 224411 (2007)

5. Kumura, T., Lashley, J.C., Tokura, Y.: Phys. Rev. Lett. 101, 220401 (2006)

6. Seki, S., Onose, Y., Tokura, Y.: Phys. Rev. Lett. 101, 067204 (2008)

7. Kimura, K., Otani, T., Nakamura, H., Wakabayashi, Y., Kimura, T.: J. Phys. Soc. Jpn. 78, 113710 (2009)

8. Kawamura, H.: J. Phys.: Condens. Matter 10, 4707 (1998)

9. Collins, M.F., Petrenko, O.A.: Can. J. Phys. 75, 605 (1997)

10. Elkhouni, T., Amami, M., Hlil, E.K., Ben Salah, A.: J. Supercond. Nov. Magn. 8, 2182 (2013)

11. Galakhov, V.R., Poteryaev, A.I., Kurmaev, E.Z., Anisimov, V.I., Bartkowski, S., Neumann, M., Lu, Z.W., Klein, B.M., Zhao, T.-R.: Phys. Rev. B 56, 4584 (1997)

12. Topfer, J., Trari, M., Gravereau, P., Chaminade, J.P., Doumerc, J.P.: Z. Kristallogr. 210, 184 (1995)

13. Doumerc, J.P., Trari, M., Topfer, J., Founes, L., Grenier, J.C., Pouchard, M., Hagenmuller, P.: Eur. J. Solid. Inorg. Chem. 31, 705 (1994)

14. Hill, N.A.: J. Phys. Chem. B 104, 6694 (2000)

15. Fiebig, M.: J. Phys. D: Appl. Phys. 38, R123 (2005) 
16. Terada, N., Nakamura, Y., Katsumata, K., Yamamoto, T., Staub, U., Kindo, K., Hagiwara, M., Tanaka, Y., Kikkawa, A., Toyama, H., Fuyuki, T., Kanmuri, R., Ishikawa, T., Kitamura, H.: J. Phys. Rev. B 74, 180315 (2006)

17. Kimura, T., Lashley, J., Ramirez, A.: vol. 74 (2006)

18. Kadowaki, H., Kikuchi, H., Ajiro, Y.: J. Phys. Condens. Matter 2, 4485 (1990)

19. Kajimoto, R., Matsuda, M., Okuda, T.: J. JAEA-Review 75, 004 (2007)

20. Damay, F., Poienar, M., Maignan, A., Rodriguez-Carvajal, J., André, G., Doumerc, J.P.: J. Phys. Rev. B 80, 094410 (2009)

21. Shannon, R.D., Rogers, D.B., Prewitt, C.T.: J. Inorg. Chem. 10, $713(1971)$

22. Tate, J., Jayaraj, M., Draeseke, A., Ulbrich, T., Sleight, A., Vanaja, K., Nagarajan, R., Wagner, J., Hoffman, R.: Thin Solid Films 411, 119 (2002)

23. Amami, M., Colin, C.V., Strobel, P., Ben Salah, A.: Physica B 406, 2182 (2011)

24. Garlea, O., Darie, C., Bougerol, C., Isnard, O., Bordet, P.: J. Sol. St. Sci. 5, 1095-1104 (2003)

25. Hebert, S., Martin, C., Maignan, A., Retoux, R., Hervieu, M., Nguyen, N., Raveau, B.: J. Phys. Rev. B 65, 104420 (2002)

26. Gao, C., Lin, F., Zhou, X., Shi, W., Liu, A.: J. Alloys Comp. 565, 154-158 (2013)

27. Dong, C.J., Yu, W.X., Xu, M., Cao, J.J., Zhang, Y., Chuai, Y.H., Wang, Y.D.: J. All. Comp. 512, 195 (2012)
28. Kizaki, H., Sato, K., Yanase, A., Katayama-Yoshida, H.: Jpn. J. Appl. Phys. 44, 1187 (2005)

29. Dietl, T., Ohno, H., Matsukura, F., Cibert, J., Ferrand, D.: Science 287, 1019 (2000)

30. Coey, J.M.D., Venkatesan, M., Fitzgerald, C.B.: Nat. Mater. 4, 173 (2005)

31. Banerjee, B.K.: Phys. Lett. 12, 16 (1964)

32. Omri, A., Tozri, A., Bejar, M., Dhahri, E., Hlil, E.K.: J. Mag. Mag. Mat. 324, 3122-3128 (2012)

33. Luo, S.J., Li, L., Wang, K.F., Li, S.Z., Dong, X.W., Yan, Z.B., Liu, J.-M.: Thin Solid Films 518, e50 (2010)

34. Khlifi, M., Tozri, A., Bejar, M., Dhahri, E., Hlil, E.K.: J. Mag. Mag. Mat. 324, 2142-2146 (2012)

35. Jlaiel, F., Amami, M., Boudjada, N., Strobel, P., Ben Salah, A.: J. Alloys Comp. 509, 7784 (2011)

36. Elkhouni, T., Amami, M., Colin, C.V., Strobel, P., Ben Salah, A.: J. Magn. Magn. Mater. 330, 101 (2013)

37. Matsui, T., Taketani, E., Fujimura, N., Ito, T., Morii, K.: J. Appl. Phys. 93, 6993 (2003)

38. Elkhouni, T., Amami, M., Colin, C.V., Strobel, P., BenSalah, A.: J. Mag. Mag. Mat. 355, 158-163 (2014)

39. Okuda, T., Kishimoto, T., Uto, K., Hokazono, T., Onose, Y., Tokura, Y., Kajimoto, R., Matsuda, M.: J. Phys. Soc. Jpn. 78, 013604 (2009)

40. Yang, Z., Ye, L., Xie, X.: J. Phys.: Condens. Matter 12, $2737-$ 2747 (2000) 